nen Betrachtungen aus, weil er nicht „schadensvorbeugend“ wirke, sondern „schadensbegrenzend“ (S. 189). Dies unterschätzt die Monitoring-Aufgabe des Stabilitätsrats und umgeht das Problem, das die OECD nennt: „In seiner derzeitigen Zusammensetzung gehören dem Rat jedoch die Finanzminister von Bund und Ländern an, so dass die Bundesregierung und die Länderregierungen sich im Wesentlichen selbst kontrollieren. Das könnte dazu führen, dass der Rat zu lax auf Haushaltsüberschreitungen oder Haushaltskosmetik reagiert." 2

Sehr überzeugend sind die Änderungsvorschläge, die der Autor entwickelt. Er plädiert für einen Wegfall der strukturellen Verschuldungsmöglichkeit des Bundes, weil er realistischerweise erwartet, dass diese immer vollständig genutzt werden wird, womit sich die Gesamtverschuldung permanent erhöht. Beim Verstoß gegen Verschuldungsregeln schlägt er einen Zuschlag zur Einkommensteuer vor, um die durch unzulässige Ausgaben entstandene Lücke zu stopfen. Hier soll der Wähler auf Regierungshandeln aufmerksam gemacht werden und an der Wahlurne Konsequenzen ziehen. Ryczewski erhofft sich auch einen Mentalitätswechsel in der Politik dahingehend, dass es nicht um „Staatsausgaben“ geht, sondern um das Geld des Steuerzahlers beziehungsweise Wählers. Hinsichtlich der Tilgungsregeln in den Ausnahmefällen, in denen eine Abweichung von den neuen Haushaltsregeln erlaubt ist (Notsituationen) wünscht sich der Autor präzisere Festlegungen, etwa eine Regelfrist von fünf Jahren. Im Augenblick ist in Artikel 115 (2) GG nur von einem „angemessenen Zeitraum" die Rede. Weiterhin diskutiert Ryczewski Vorschläge, den Kreis möglicher Klageberechtigter beim Bundesverfassungsgericht zu erweitern. Er präferiert ein Antragsrecht für die Parlamentsfraktionen gegenüber einem solchen für den Bundesrechnungshof, der zwar fachlich kompetent aber demokratisch schwächer legitimiert sei. In der Summe ist Christoph Ryczewski zu bescheinigen, dass ihm ein sehr kluger Beitrag zum Thema „Schuldenbremse" gelungen ist.

Roland Sturm

2 Ebenda, S. 73.

\title{
Deutschland und Europa: weiter Blick zu Ehren von William Paterson
}

Bulmer, Simon, Charlie Jeffery und Stephen Padgett (Hrsg.): Rethinking Germany and Europe. Democracy and Diplomacy in a Semi-Sovereign State, Palgrave Macmillan, Houndsmills 2010, 243 Seiten, $€ 71,99$.

Die britische Politikwissenschaft hat in den letzten Jahren und Jahrzehnten die Einbindung der Bundesrepublik in die europäischen Strukturen intensiv begleitet. Zu deren exponiertesten Autoren gehört zweifellos William Paterson, um dessen Wirken sich der angezeigte Band dreht. Das von Simon Bulmer, Charlie Jeffery und Stephen Padgett - drei weiteren Exponenten der britischen Deutschlandforschung - edierte Werk stellt eine etwas ungewöhnliche Festschrift dar, wie die Herausgeber in ihrer Einleitung selbst anmerken (S. 2). Viele der hier versammelten Texte rekurrieren direkt auf Paterson, allerdings häufig eher am Ran- 
de. Die Themensetzung ist von der beeindruckenden Bandbreite inspiriert, die Paterson in seiner fast vier Jahrzehnte währenden Publikationstätigkeit abgedeckt hat.

In ihrer Einleitung schlagen die drei Herausgeber zwei Schneisen, entlang derer sich die insgesamt zwölf Beiträge des Bandes bewegen. Die erste verfolgt die Frage der Kontinuität der deutschen Politik gegenüber Europa. Die Autoren überraschen mit einer Phaseneinteilung, die mit den Jahren 1969 und 1998 lediglich zwei Zäsuren in der deutschen Europapolitik setzt. Weder der Mauerfall noch die Wiedervereinigung werden demnach als epochal angesehen. Die schwer zu bewältigenden Folgen der Wiedervereinigung haben allerdings, so die Autoren, zu einer Neutarierung des Verhältnisses von Deutschland und Europa geführt: „The EU now (...) acts as much or more as a source of constraint on Germany than a milieu in which German actors can feel at home." (S. 8)

Die Beschränkung der deutschen Politik durch Europa wird zweitens an drei Problembereichen durchbuchstabiert: Diskutiert werden der Niedergang der Parteiendemokratie, die zunehmende Fragmentierung der Institutionen im sozial- und wirtschaftspolitischen Modell sowie der Verlust alter Sicherheiten in der internationalen Kooperation. In der Summe erschweren diese Probleme nach Bulmer, Jeffery und Padgett vor allem die Fähigkeit des deutschen Regimes, adäquate politische Führung zu generieren: „Germany suffers from a leadership deficit." (S. 20)

Die weiteren Texte des Sammelbandes bewegen sich zwischen einem pessimistischen und optimistischen Pol. Die pessimistische Variante sieht Deutschland auf breiter Front im Niedergang; viele Texte verweisen auf das einschlägige Sonderheft der Zeitschrift West European Politics aus dem Jahre 2003 (herausgegeben von Herbert Kitschelt und Wolfgang Streeck), das die These „von der Stabilität zur Stagnation“ formulierte. Einige Autoren sehen die Dinge indes weniger dramatisch. Simon Green kommt in einem Text zum Thema „Economic Governance in Germany" zu dem Schluss, dass es insgesamt eine deutliche Heterogenisierung des Modells des rheinischen Kapitalismus gegeben habe. Diese habe zu einer stärkeren gesellschaftlichen Individualisierung und daher zu einer kulturell fundierten Aufkündigung des konsensualen Modells geführt (S. 96). Wyn Grant, der sich den Industriebeziehungen zuwendet, bringt den bei Green impliziten Vergleich auf den Punkt. Entscheidend sei, ob die Leistung des semi-souveränen Modells in Bezug zur alten Bundesrepublik oder zu anderen Industriestaaten gesetzt werde. Geschehe letzteres, sei die semi-souveräne Bundesrepublik nach wie vor zu den wettbewerbsfähigen Regimen zu zählen (S. 108). In diese Linie passt auch der Befund von Manfred G. Schmidt, der trotz der erschwerten Bedingungen seit 1990 von einem Fortbestand der „Politik des mittleren Weges" ausgeht (S. 82).

Die bisher angezeigten Beiträge des Sammelbandes werden - zusammen mit weiteren Schriften, auf die hier aus Platzmangel nicht eingegangen werden kann - im ersten Teil des Buches abgedruckt, der mit „Democracy and Governance in Germany" betitelt ist. Europäische Bezüge werden hier nicht systematisch, sondern in der Tat eher als Randbedingungen für politisches Handeln in der Bundesrepublik thematisiert. Dies ändert sich in den sechs Beiträgen des zweiten Buchteils: „European Contexts“. Hier behandeln Simon Bulmer, Charlie Jeffery, William Chandler, Hans Maull und weitere Autoren unterschiedliche internationale Zusammenhänge, wobei transatlantischen und globalen Aspekten breiter Raum gegeben wird.

In einem instruktiven Beitrag beschreibt Emil Kirchner die Rolle der Bundesrepublik in der internationalen Sicherheitspolitik. Deutlich wird dabei, dass sich die deutsche Zurück- 
haltung hinsichtlich eines robusten sicherheitspolitischen Engagements einerseits bis in die Gegenwart fortgesetzt hat. Andererseits stellt die Bundesrepublik überdurchschnittlich viel Personal in militärischen Missionen und investiert mehr als viele andere EU-Staaten in europäische Entwicklungspolitik (S. 147 - 150). Insofern ist Deutschland als eine der treibenden Kräfte zu sehen, die den Charakter der EU als Zivilmacht prägen, wie Hanns Maull ausführt. Diese These ist allerdings Maulls im Buch abgedruckten Text zur „EU als globaler Zivilmacht" (S. 200 - 220) nur implizit zu entnehmen - Deutschland kommt darin nur ganz am Rande vor.

Instruktiver für das Thema des Bandes sind drei Beiträge, in denen das Verhältnis der Bundesrepublik zu den wichtigen außenpolitischen Partnern Frankreich (William Chandler), USA (James Sperling) und Großbritannien (Alister Miskimmon und John Roper) untersucht wird. Die deutsch-französischen Beziehungen werden dabei unter dem Fokus des Verhältnisses von Angela Merkel und Nicolas Sarkozy abgehandelt. Die Darstellung Chandlers erweist sich als differenziert und reichhaltig. Dennoch kann angezweifelt werden, ob die Konzentration auf Kanzlerin und Präsident den vielschichtigen Beziehungen zwischen Deutschland und Frankreich in vollem Umfang gerecht wird. Im Verhältnis zu den USA verwendet James Sperling die Gulliver-Metapher, um das Missverhältnis zwischen starker ökonomischer und geringer politischer Macht der Bundesrepublik zu charakterisieren (S. 182). Sperlings Diagnose widerspricht der pessimistischen Linie der Deutschlandforschung, insofern Deutschland als „entfesselter“ Gulliver angesehen wird, der sich von amerikanischem Einfluss weitgehend emanzipiert hat (S. 186).

Insgesamt präsentieren Bulmer, Jeffery und Padgett eine Reihe lesenswerter Analysen der deutschen Politik gut zwanzig Jahre nach dem Fall der Berliner Mauer. Die Einbindung der Bundesrepublik in europäische Strukturen wird ihrerseits kontextualisiert und damit um eine internationale Komponente angereichert, die den Blick wohltuend weitet.

Timm Beichelt

\section{Frankreichs Geschichte: Nutzen der Vergangenheit gelungen diskutiert}

Deutsch-Französisches Institut (Hrsg.): Frankreich Jahrbuch 2010. Frankreichs Geschichte: Vom (politischen) Nutzen der Vergangenheit, VS-Verlag für Sozialwissenschaften, Wiesbaden 2011, 296 Seiten, $€ 39,95$.

Seit 1988 wird jährlich vom Deutsch-Französischen Institut in Ludwigsburg das Frankreich Jahrbuch ediert. Die mittlerweile 23 Bände bieten allen am Nachbarland Interessierten vorzügliche Einblicke in aktuelle politische Entwicklungen, in wirtschaftliche sowie soziale Problemfelder sowie in zahlreiche kulturelle Bereiche. Umrahmt werden die Schwerpunktbeiträge von einer bestens recherchierten Dokumentation über gesellschaftliche und sozioökonomische Basisdaten, einer Chronik mit den wichtigsten Begebenheiten im Berichtszeitraum, Wahlergebnissen sowie - im Falle einer Kabinettsumbildung - Angaben zur Zusammensetzung der neuen Regierung. Hinzu kommt jeweils eine umfangreiche, klar nach Themenfeldern gegliederte deutschsprachige Literaturübersicht zu Frankreich. Kurz: Die Jahrbücher zählen zum unverzichtbaren „Handwerkszeug“ für Frankreichforscher, aber 